\title{
Effect of the microtubule-associated protein tau on dynamics of single-headed motor proteins KIF1A
}

\author{
J. Sparacino, ${ }^{1, *}$ M. G. Farías, ${ }^{2}$ and P. W. Lamberti ${ }^{1}$ \\ ${ }^{1}$ Facultad de Matemática, Astronomía y Física, Universidad Nacional de Córdoba and CONICET, Medina Allende s/n, \\ Ciudad Universitaria, 5000 Córdoba, Argentina \\ ${ }^{2}$ Instituto de Investigación Médica Mercedes y Martín Ferreyra, INIMEC-CONICET and FoNCyT, Friuli 2434, \\ Barrio Parque Vélez Sarsfield, 5016 Córdoba, Argentina \\ (Received 20 November 2012; published 19 February 2014)
}

\begin{abstract}
Intracellular transport based on molecular motors and its regulation are crucial to the functioning of cells. Filamentary tracks of the cells are abundantly decorated with nonmotile microtubule-associated proteins, such as tau. Motivated by experiments on kinesin-tau interactions [Dixit et al., Science 319, 1086 (2008)] we developed a stochastic model of interacting single-headed motor proteins KIF1A that also takes into account the interactions between motor proteins and tau molecules. Our model reproduces experimental observations and predicts significant effects of tau on bound time and run length which suggest an important role of tau in regulation of kinesin-based transport.
\end{abstract}

DOI: 10.1103/PhysRevE.89.022714

PACS number(s): 87.16.Nn, 05.40.-a, 45.70.Vn, 87.16.Wd

\section{INTRODUCTION}

Intracellular transport is fundamental for cellular function, survival, and morphogenesis. Kinesin superfamily proteins (also known as KIFs) are important molecular motors that directionally transport cargoes along microtubules (MTs), including membranous organelles, protein complexes, and messenger ribonucleic acids (mRNAs) [1]. Disruptions or defects of MT-based transport are observed in many neurodegenerative diseases [2,3]. MTs are decorated with nonmotile microtubule-associated proteins (MAPs) that promote MT assembly and play important roles in organizing the MT cytoskeleton [4,5]. Kinesin proteins interfere with MAPs, being the latest able to inhibit active transport of cytoplasmic material. Kinesin competes with MAPs to bind to the MT surface, and MAPs bound to MTs might also block the path of motor proteins. Tau is a mainly neuronal MAP, enriched in the axonal compartment [6] that has been shown to inhibit plus end -directed transport of vesicles along MTs by kinesin [7]. Tau reduces not only the attachment frequency of kinesin to MTs but also the distance that kinesin travels along the MT in a single run [8]. It is also known that when single kinesin motors encounter tau patches on the MT, most of the motors detach from the MT surface [9].

Some recent theoretical models of interacting molecular motors [10-13] are extensions of asymmetric simple exclusion processes (ASEPs) [14] in which motors are represented by particles that hop along a one-dimensional lattice with hard-core exclusion. A paper by Parmeggiani et al. [15] introduced an ASEP-like model that relaxes the restriction of the conservation of particles in the bulk, allowing the attachment or detachment of particles in any site. In this paper we consider specifically the effect of MAP tau in the intracellular traffic of single-headed kinesin motor protein KIF1A [16]. A model for KIF1A motor transport has been proposed by Nishinari et al. [17] that enriched Parmeggiani's model via two significant modifications. First, the model explicitly incorporated the Brownian ratchet mechanism for

\footnotetext{
*sparacin@famaf.unc.edu.ar
}

individual KIF1A motors, i.e., the mechanochemical coupling involved in their directed motion, including the adenosine triphosphate (ATP) hydrolysis that fuels the cyclic steps of the motor. Second, the model included a construction based on parameters that have direct correspondence with experimentally controllable quantities, of which the two most important are ATP and motor concentrations. There are several types of models for describing the dynamics of molecular motors intracellular transport. On one hand, Refs. [10-13,15,17,18] consider only motor-motor interactions. On the other hand, models including lattice defects have been recently presented [19-21]. For example, Chai et al. [20] consider transport of molecular motors in the presence of static defects, and Turci et al. [21] study the transport along a lattice with dynamical defects. Here we investigate the effect of the MAP tau in kinesin dynamics via parameters that are related to experimentally observed quantities by Dixit $e t a l$. [9].

\section{LATTICE-GAS MODEL FOR KIF1A DYNAMICS IN PRESENCE OF TAU MOLECULES}

We work with a one-dimensional lattice of $L$ sites that represents one MT's protofilament. Each site on the lattice corresponds to one motor-binding site on the protofilament. The lattice parameter is taken to be $8 \mathrm{~nm}$, which is the separation between adjacent binding sites of the MT. Each KIF1A is represented by a particle that, when bound to one site of the filament, can hop to any of the two nearest-neighbor sites. The motor protein can also attach to (detach from) the MT; this is modeled as a particle creation (annihilation) on the filament. Each site of the filament has two internal states that represent the two possible bound states in which a KIF1A motor can be related to the MT. In state 1, the motor protein is strongly bound to the MT, whereas in state 2 it is weakly bound (the motor protein is tethered to the MT by an electrostatic attraction that prevents it from diffusing away from the filament) [16]. Therefore, there are three possible states for each site: 0 if the site is empty, 1 if the site is occupied with a motor in state 1 , and 2 if the site is occupied with a motor in state 2 . We consider a distribution of tau molecules decorating the filament. Each binding site of the 

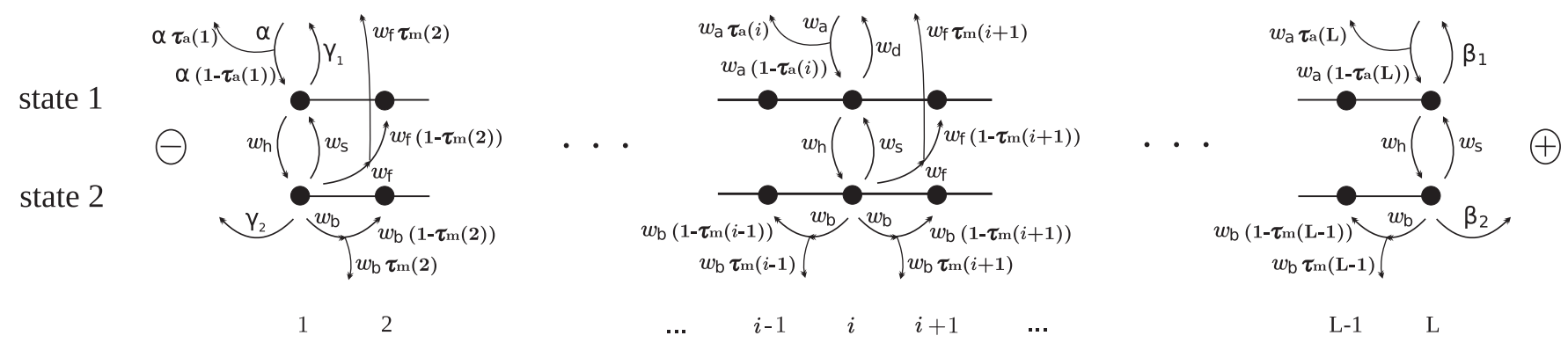

FIG. 1. Scheme of the model showing the updating possibilities and the associated transition rates for a bulk site, as well as for the minus and plus ends. The effect of tau, preventing a motor attachment or forcing a moving motor to detach, is graphically depicted as branching arrows. See main text for more details.

filament can either have one tau molecule or no molecule at all. To characterize the amount of tau present in the MT, we will refer to the concentration of tau molecules, denoted by $C_{\text {tau }}$, defined as the number of tau molecules per site in the filament. The tau concentration therefore ranges between 0 and 1, with these extreme cases representing no tau molecules attached to any site of the MT and a tau molecule attached to every site of the filament, respectively.

The dynamical evolution of the system is described with transition rates that reflect the stochastic nature of the movement of the motor protein and of its interaction with tau molecules. The change of state of a given site depends on the state of occupation of the site, the state of occupation of its nearest-neighbor sites, and whether or not there are tau molecules in the sites under consideration. We run Monte Carlo simulations that update the state of all sites of the filament following the random sequential method. In one Monte Carlo step (MCS) we change the state of occupation of all sites in a random order.

Figure 1 shows a scheme with the updating possibilities for a site in the bulk and for the two ends of the lattice.

Let us consider the $i t h$ site: if the site is empty, the only possible state change is that a motor binds to it in state 1 , with a rate $\omega_{a}\left[1-\tau_{a}(i)\right] . \tau_{a}(j)$ represents the probability that a tau molecule prevents the attachment of a KIF1A motor protein to site $j$. It is equal to 0 if there is no tau molecule in the site, and equal to $p_{a}$ if there is a tau molecule in the site. If the site $i$ is occupied with a motor in state 1 , two things can happen. First, the motor protein can detach from the site, with a rate $\omega_{d}$, and second, the motor protein can hydrolyze the ATP molecule and consequently change to state 2 , with a rate $\omega_{h}$. If the site is occupied with a motor in state 2 , several possibilities may arise. The motor protein can diffuse to one of its nearest-neighbor sites with a rate $\omega_{b}\left[1-\tau_{m}(i-1)\right]$ towards the minus end, and $\omega_{b}\left[1-\tau_{m}(i+1)\right]$ towards the plus end (staying in state 2$)$; it can release adenosine diphosphate (ADP) and move forward with a rate $\omega_{f}\left[1-\tau_{m}(i+1)\right]$ (binding to the next site in state $1)$, or, finally, it can release ADP and stay in the same site with a rate $\omega_{s}$ (changing to state 1$) . \tau_{m}(j)$ represents the probability that a tau molecule in site $j$ forces a moving motor protein to detach. It is equal to 0 if there is no tau molecule in the site, and equal to $p_{m}$ if there is a tau molecule in the site. It is important to emphasize that over the course of the updating process a change of site is possible only if the target site is empty.
The two ends of the lattice deserve special consideration, we take $\alpha$ and $\delta$ instead of $\omega_{a}$ as the attachment rates for the minus and plus ends, respectively. In the same way, $\gamma_{1}$ and $\beta_{1}$ (instead of $\omega_{d}$ ) are the detachment rates, and $\gamma_{2}$ and $\beta_{2}$ (instead of $\left.\omega_{b}\right)$ are the exit rates of the motors due to Brownian motion for the minus and plus ends, respectively.

Rate $\omega$ means that in an infinitesimal time interval $d t$, the probability of the event occurring is $\omega d t$.

The two ways in which kinesin interacts with tau molecules are schematically shown in Fig. 2. Our model captures the essence of the experimental results on tau-kinesin interactions [9], which can be summarized as the following: (1) the frequency of kinesin binding to MT depends on local tau concentration; (2) the average binding frequency of kinesin in the presence of tau in the cytosol reduces to approximately one-third of the value without tau; and (3) approximately half of the times that a kinesin motor protein encounters a tau molecule, it detaches from the MT, and the remaining times it passes or pauses. Therefore, the probability that a tau molecule prevents a kinesin motor protein binding to the MT is taken to be $p_{a}=0.67$, and the probability that a tau molecule forces a moving kinesin motor protein to detach from the MT is chosen to be $p_{m}=0.5$.

All parameters that have no relation with tau were chosen following Ref. [17]. For our purposes, the two most important parameters are the hydrolysis rate $\omega_{h}$, and the binding rate $\omega_{a}$.

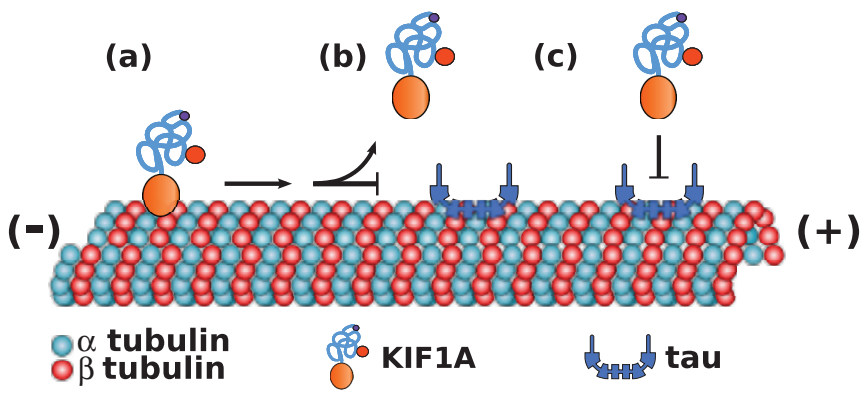

FIG. 2. (Color online) Cartoon illustrating the interaction between KIF1A and tau. (a) A KIF1A motor protein binds to a tau-free site and moves along sites with no tau molecules in normal fashion. (b) A tau molecule bound to the MT may force a moving KIF1 A motor to detach from the filament upon an encounter. (c) A tau molecule bound to a particular site of the MT may prevent a KIF1A motor protein from attaching to the filament in that site. See main text for more details. 
It is worth remembering that $\omega_{h}$ is a monotonous increasing function of the ATP concentration, ranging between 0 (when there are no ATP molecules in the cytosol) and $0.25 \mathrm{~ms}^{-1}$ (for saturating ATP concentration). Moreover, $\omega_{a}$ is proportional to the KIF1A concentration: $\omega_{a}=10^{-2} C_{\mathrm{KIF} 1 \mathrm{~A}} \mathrm{nM}^{-1} \mathrm{~s}^{-1}$.

\section{A. Mean-field equations}

Let us denote by $r_{i}$ and $q_{i}$ the probabilities of finding a KIF1A motor in states 1 and 2, respectively, at the lattice site $i$ at time $t$. According to the stated in the previous section, the master equations for the dynamics of motors in a bulk site are given by the following:

$$
\begin{aligned}
\frac{d r_{i}}{d t}= & {\left[1-\tau_{a}(i)\right] \omega_{a}\left(1-r_{i}-q_{i}\right)-\left(\omega_{d}+\omega_{h}\right) r_{i}+\omega_{s} q_{i} } \\
+ & {\left[1-\tau_{m}(i)\right] \omega_{f} q_{i-1}\left(1-r_{i}-q_{i}\right) } \\
\frac{d q_{i}}{d t}= & \omega_{h} r_{i}-\omega_{s} q_{i}-\omega_{f} q_{i}\left(1-r_{i+1}-q_{i+1}\right) \\
& -\omega_{b} q_{i}\left(2-r_{i-1}-q_{i-1}-r_{i+1}-q_{i+1}\right) \\
& +\left[1-\tau_{m}(i)\right] \omega_{b}\left(q_{i-1}+q_{i+1}\right)\left(1-r_{i}-q_{i}\right)
\end{aligned}
$$

where

$$
\tau_{a}(i)=\left\{\begin{array}{ll}
p_{a} & \text { if there is a tau molecule on site } \mathrm{i} \\
0 & \text { if there is not a tau molecule on site } \mathrm{i}
\end{array},\right.
$$

and

$$
\tau_{m}(i)=\left\{\begin{array}{ll}
p_{m} & \text { if there is a tau molecule on site } \mathrm{i} \\
0 & \text { if there is not a tau molecule on site } \mathrm{i}
\end{array} .\right.
$$

The probability of site $i$ being empty at time $t$ is given by $1-r_{i}(t)-q_{i}(t)$. The corresponding equations for the minus end are:

$$
\frac{d r_{1}}{d t}=\left[1-\tau_{a}(1)\right] \alpha\left(1-r_{1}-q_{1}\right)-\left(\gamma_{1}+\omega_{h}\right) r_{1}+\omega_{s} q_{1},
$$

$$
\begin{aligned}
\frac{d q_{1}}{d t}= & \omega_{h} r_{1}-\omega_{s} q_{1}-\omega_{f} q_{1}\left(1-r_{2}-q_{2}\right) \\
& -\omega_{b} q_{1}\left(1-r_{2}-q_{2}\right)-\gamma_{2} q_{1} \\
& +\left[1-\tau_{m}(1)\right] \omega_{b} q_{2}\left(1-r_{1}-q_{1}\right),
\end{aligned}
$$

and for the plus end:

$$
\begin{aligned}
\frac{d r_{L}}{d t}= & {\left[1-\tau_{a}(L)\right] \delta\left(1-r_{L}-q_{L}\right)-\left(\beta_{1}+\omega_{h}\right) r_{L}+\omega_{s} q_{L} } \\
& +\left[1-\tau_{m}(L)\right] \omega_{f} q_{L-1}\left(1-r_{L}-q_{L}\right) \\
\frac{d q_{L}}{d t}= & \omega_{h} r_{L}-\omega_{s} q_{L}-\beta_{2} q_{L}-\omega_{b} q_{L}\left(1-r_{L-1}-q_{L-1}\right) \\
& +\left[1-\tau_{m}(L)\right] \omega_{b} q_{L-1}\left(1-r_{L}-q_{L}\right) ;
\end{aligned}
$$

$\tau_{a}(1)$ and $\tau_{a}(L)$ are defined as in Eq. (3), and $\tau_{m}(1)$ and $\tau_{m}(L)$ are defined as in Eq. (4).

\section{B. High-density limit}

We obtained an analytical approximate solution that allows us to test our simulations in the high motor concentration limit. In order to compute that solution we assumed periodic boundary conditions. A system with periodic boundary conditions is not realistic. However, in the high motor concentration limit, it provides a good approximation for a system with the same value for the parameters but with open boundary conditions. This is the case because in the high motor concentration limit there is a jammed region that covers the whole MT, making every site indistinguishable from the point of view of its state of occupation [22]. Moreover, we take $\tau_{a}(i)=p_{a} C_{\text {tau }} \equiv \tilde{\tau}_{a}$ and $\tau_{m}(i)=p_{m} C_{\text {tau }} \equiv \tilde{\tau}_{m}$. This corresponds to considering a tau molecule attached to every site (which, again, allows us to treat every site as indistinguishable) but with a reduced effect according to the total tau concentration in the filament.

With those assumptions, the solutions $\left(r_{i}, q_{i}\right)=(r, q)$ of the mean-field equations in the steady state are found to be

$$
\begin{gathered}
r=\frac{1+\left[K_{s}\left(1-\Omega_{f}\right)-1\right] q}{1+K_{d}+K_{h}\left(1-\Omega_{f}\right)} \\
q=\frac{-A-\sqrt{A^{2}-4 \Omega_{h}\left[K_{d}+\left(K_{h}+K_{s}\right)\left(1-\Omega_{f}\right)\right]}}{2\left[K_{d}+\left(K_{h}+K_{s}\right)\left(1-\Omega_{f}\right)\right]},
\end{gathered}
$$

where $A=K_{h}\left(\Omega_{f}-1\right)-K_{d}\left(\Omega_{s}+1\right)-\left(\Omega_{s}+\Omega_{h}\right)$,

$$
\begin{aligned}
& K_{s}=\frac{\omega_{s}}{\left(1-\tilde{\tau}_{a}\right) \omega_{a}}, \quad K_{h}=\frac{\omega_{h}}{\left(1-\tilde{\tau}_{a}\right) \omega_{a}}, \quad K_{d}=\frac{\omega_{s}}{\left(1-\tilde{\tau}_{a}\right) \omega_{a}}, \\
& \Omega_{s}=\frac{\omega_{s}}{\omega_{f}+2 \omega_{b} \tilde{\tau}_{m}}, \quad \Omega_{h}=\frac{\omega_{h}}{\omega_{f}+2 \omega_{b} \tilde{\tau}_{m}}, \quad \text { and } \\
& \Omega_{f}=\frac{\left(1-\tilde{\tau}_{m}\right) \omega_{f}}{\omega_{f}+2 \omega_{b} \tilde{\tau}_{m}} .
\end{aligned}
$$

Figure 3 shows a comparison between these analytical expressions and numerical simulations for three cases corresponding to different magnitudes of the tau-kinesin interaction. The agreement is good, and it is better for lower strengths of the tau-kinesin interaction, that is, for smaller values of the parameters $p_{a}$ and $p_{m}$. This is consistent with the fact that the assumption of indistinguishable sites made in the analytical approximate solutions is true for the simulations whenever the tau-kinesin interaction is low enough for not introducing local differences between sites. However, in real systems as well as in our simulations, a site has or has not an attached tau molecule (there are no intermediates states), so the assumption is true only for no tau-kinesin interaction.

The limit with no tau molecules present in the cytosol can be obtained taking $\tilde{\tau}_{a}=0$ and $\tilde{\tau}_{m}=0$. In that case, expressions (9) and (10) reduce to the ones found by Nishinari et al. in the model for KIF1A dynamics without tau molecules [17].

\section{BOUND TIMES AND RUN LENGTHS}

We work with a MCS representing $0.71 \mathrm{~ms}$ of real time. Every simulation has a fixed concentration of tau molecules and starts with no motor attached to the filament. In order to construct the distribution of tau molecules with the desired concentration, we give to each site of the filament the chance of having a tau molecule with probability $C_{\text {tau }}$. Dixit et al. found that tau patches were stable over the time course of several minutes while the average duration of motor protein runs are on the order of seconds or tens of seconds [9]. Therefore, the tau distribution is held constant during the complete course of a simulation. 
(a)

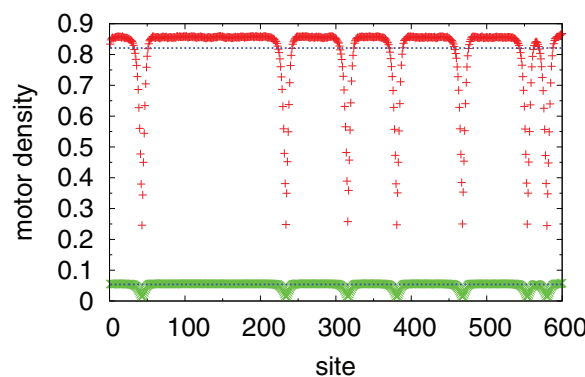

(b)

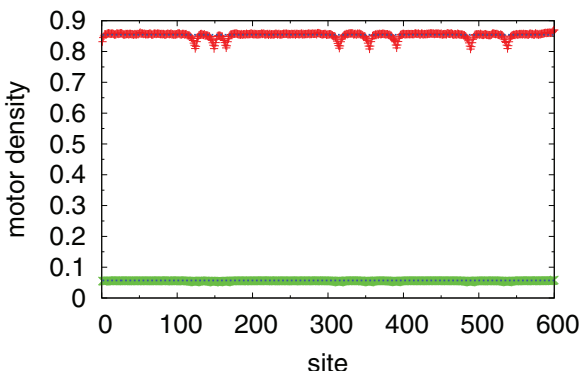

(c)

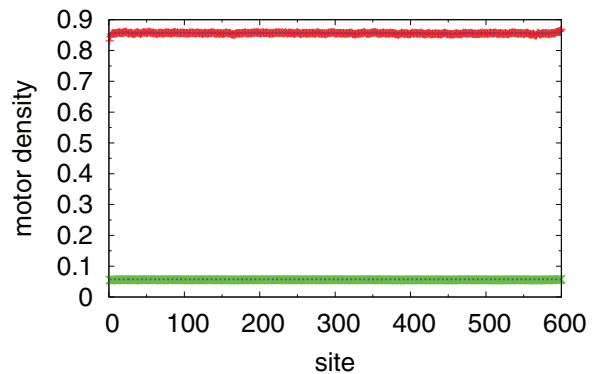

FIG. 3. (Color online) Stationary motor density profiles. The red upper (green lower) points correspond to numerical results for the state 1 (state 2) motor density with open boundary conditions. The dashed lines are the analytical approximate predictions for periodic systems with the same parameters. The strength of the tau-kinesin interaction is given by the parameters $p_{a}=0.67, p_{m}=0.5$ (left), $p_{a}=0.067$, $p_{m}=0.05$ (center), and $p_{a}=0.0067, p_{m}=0.005$ (right). Model parameters are $L=600, C_{\text {tau }}=0.01, \omega_{a}=\alpha=0.001\left[\mathrm{~ms}^{-1}\right], \omega_{d}=\beta_{1}=$ $\beta_{2}=0.0001\left[\mathrm{~ms}^{-1}\right], \omega_{s}=0.145\left[\mathrm{~ms}^{-1}\right], \omega_{f}=0.055\left[\mathrm{~ms}^{-1}\right], \omega_{b}=0.6\left[\mathrm{~ms}^{-1}\right], \omega_{h}=0.01\left[\mathrm{~ms}^{-1}\right]$ and $\gamma_{1}=\gamma_{2}=\delta=0$. Note that the chosen $\omega_{a}$ value allows us to realize the high-density limit [22].

In each run of the simulation the bound time is calculated by averaging the intervals between attachment and detachment of each KIF1A. Similarly, in each simulation the run length is calculated by averaging the distances that each motor travels

(a)

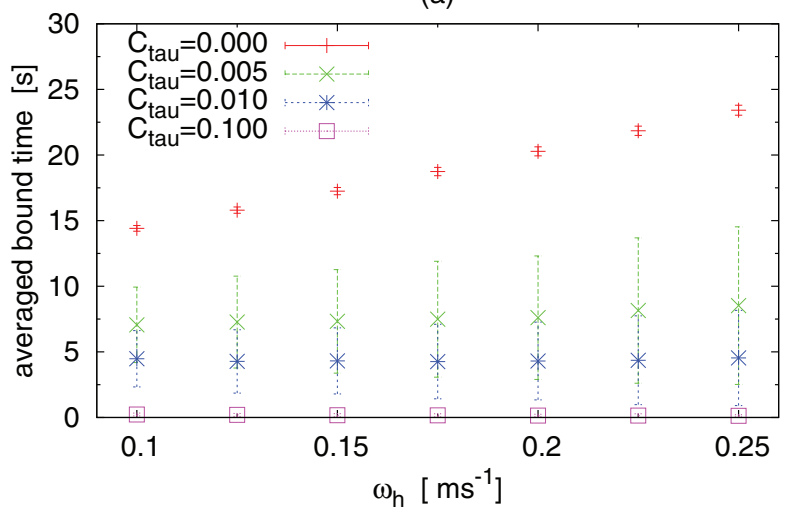

(b)

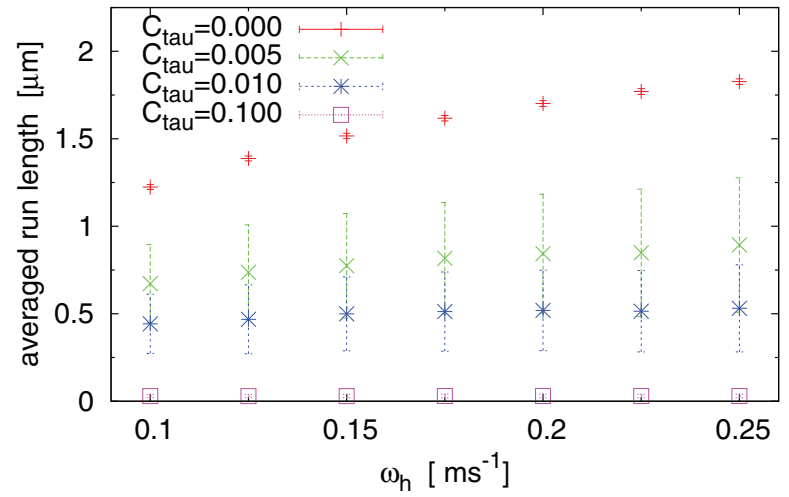

FIG. 4. (Color online) Low motor density case. (a) Averaged time between attachment and detachment of motor proteins on the filament as a function of the hydrolysis rate. (b) Average length spanned by KIF1A motors on the filament in single runs as a function of the hydrolysis rate. Model parameters are $L=$ 600, $p_{a}=0.67, p_{m}=0.5, \omega_{a}=\alpha=0.00001\left[\mathrm{~ms}^{-1}\right], \omega_{d}=\beta_{1}=$ $\beta_{2}=0.0001\left[\mathrm{~ms}^{-1}\right], \omega_{s}=0.145\left[\mathrm{~ms}^{-1}\right], \omega_{f}=0.055\left[\mathrm{~ms}^{-1}\right], \omega_{b}=$ $0.6\left[\mathrm{~ms}^{-1}\right]$, and $\gamma_{1}=\gamma_{2}=\delta=0$. between attachment and detachment. The observables shown in Figs. 4 and 5 were obtained averaging the results over 1000 simulations with the same parameters except for the random number generator seed, i.e., the observables shown are

(a)

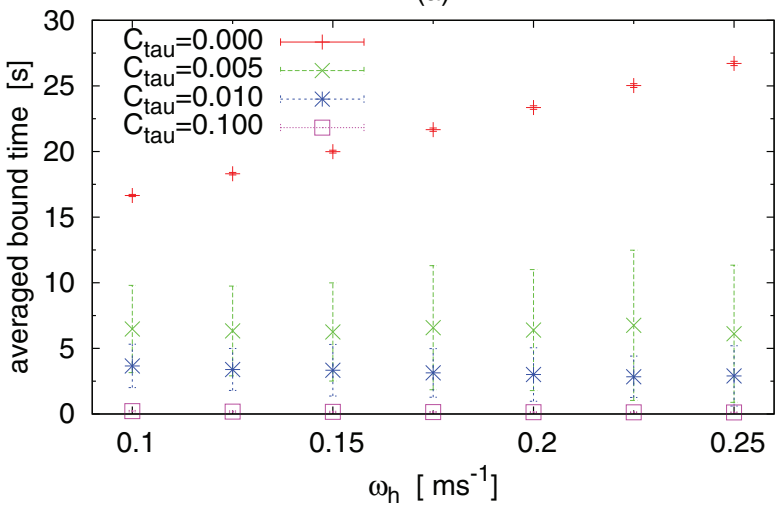

(b)

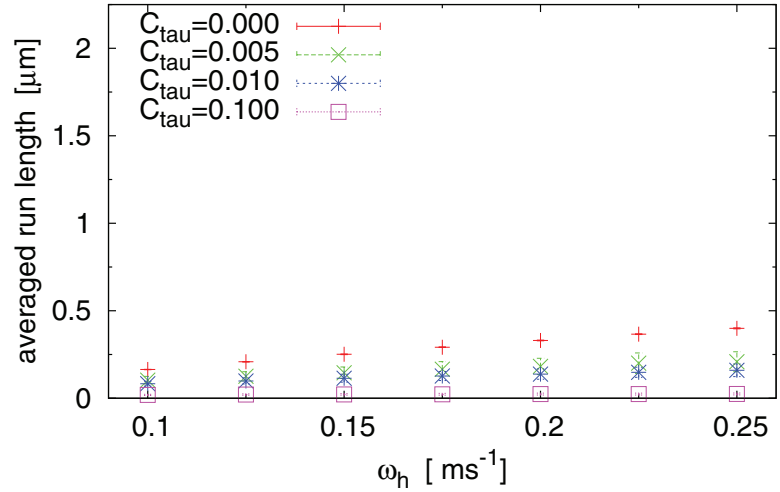

FIG. 5. (Color online) High motor density case. (a) Averaged time between attachment and detachment of motor proteins on the filament as a function of the hydrolysis rate. (b) Average length spanned by KIF1A motors on the filament in single runs as a function of the hydrolysis rate. Model parameters are $L=600, p_{a}=0.67$, $p_{m}=0.5, \omega_{a}=\alpha=0.001\left[\mathrm{~ms}^{-1}\right], \omega_{d}=\beta_{1}=\beta_{2}=0.0001\left[\mathrm{~ms}^{-1}\right]$, $\omega_{s}=0.145\left[\mathrm{~ms}^{-1}\right], \omega_{f}=0.055\left[\mathrm{~ms}^{-1}\right], \omega_{b}=0.6\left[\mathrm{~ms}^{-1}\right]$, and $\gamma_{1}=$ $\gamma_{2}=\delta=0$. 
averages of averages. The error associated with the observables corresponds to the standard deviation. It is important to emphasize that, although the tau distribution is held constant in the course of a simulation, each of the 1000 simulations generates a new tau distribution (with the same concentration) and evolves for $2 \times 10^{6}$ MCSs after a thermalization of $2 \times 10^{5} \mathrm{MCSs}$, which guarantees that the system reaches the stationary state.

Figures 4 and 5 show the bound time and the run length as a function of $\omega_{h}$ for a low motor density case and a high motor density case, respectively.

In Fig. 4(a), when there are no tau molecules present in the cytosol $\left(C_{\text {tau }}=0\right)$ the bound time grows with $\omega_{h}$ from near $15 \mathrm{~s}$ for low ATP concentration to almost $25 \mathrm{~s}$ for saturating ATP concentration. When there are no tau molecules bound to the MT, motor proteins can detach from the filament only when they are in state 1 . So, as $\omega_{h}$ increases, the ATP concentration increases and the time that a motor is in state 2 becomes larger (if there are enough ATP molecules, the motor hydrolyzes one and changes to state 2 almost immediately). Thereby, the time duration of the motor runs in the MT increases. On the other hand, in the presence of tau molecules, KIF1A motors can be forced to detach even if they are in state 2 . When a moving motor protein (either actively moving forward or diffusively moving towards either end) encounters a tau molecule in its target site, it is forced to detach from MT with a probability $p_{m}$. In this way the presence of tau molecules shortens the averaged bound time. The greater the values of tau concentration, $C_{\mathrm{tau}}$, the larger the number of KIF1A-tau encounters and the stronger the effect of tau on the averaged bound time. This can be observed in Fig. 4(a), where the values of averaged bound time are ordered with increasing value of $C_{\text {tau }}$ from top to bottom. The results for $C_{\text {tau }}=0.005$, in which the tau concentration is very low, are similar to the case without tau and can be understood in a similar way. For $C_{\mathrm{tau}}=0.01$ and $C_{\mathrm{tau}}=0.1$ the tau concentration is large enough to cancel the effect of the high ATP concentration, and the averaged bound time remains almost constant for all $\omega_{h}$ values. Moreover the averaged bound time for $C_{\text {tau }}=0.1$ presents a slightly decreasing tendency with increasing values of $\omega_{h}$ (averaged bound time decreases approximately from
0.22 to $0.13 \mathrm{~s}$ ), which corresponds to a tau-ruled behavior. A high ATP concentration accelerates the dynamic of the motors favoring the encounters with tau molecules and increasing the chances of tau-mediated detachment.

The effect of tau on KIF1A dynamics for the low motor density case considering the averaged run length can be established in similar terms. First, we can note in Fig. 4(b) that in absence of tau molecules the averaged run length increases with $\omega_{h}$. The ATP molecules are the fuel that KIF1A motors use to actively move toward the plus end of MT, so that a greater ATP concentration accelerates the dynamic of the motors, enlarging the distance that the motors travel before detaching from MT. In the presence of tau molecules, the movement of KIF1A along the MT can give room to encounters with tau molecules so that a competition begins when we increase the ATP concentration. On the one hand, as we already mentioned, the availability of ATP molecules favors longer motor run lengths, but, on the other hand, it also favors larger numbers of kinesin-tau encounters, which shortens motor run lengths. Therefore for $C_{\mathrm{tau}}=$ 0.005 and $C_{\mathrm{tau}}=0.01$ the run lengths are still increasing functions of $\omega_{h}$, but for $C_{\text {tau }}=0.1$ it remains almost constant for the whole range of $\omega_{h}$. In this case, the tau concentration is large enough to compensate the higher ATP concentration with a greater number of motor detachments. For the same value of $\omega_{h}$ (the same ATP concentration) the run length is always smaller for larger values of $C_{\text {tau }}$; that is, the effect of tau is to shorten the distance that the motors travel along the MT.

Unlike the described situation, in the high motor density case, the filament is completely jammed and the movement of the motors is severely limited. This is why the bound time values remain almost the same but the run length values change significantly, as can be seen in Figs. 5(a) and 5(b), respectively. When a motor is already bound to a site of the filament and remains immobile, tau cannot force it to detach. Thus, as a consequence of the reduced motility, the bound time values are slightly greater than those corresponding to the low motor density case. On the other hand, the run length values show a significant decrease compared to the low-density case, which is a joint effect of both the jamming that limits the motors motility and tau-kinesin interactions that favors the unbinding of the motors. (a)

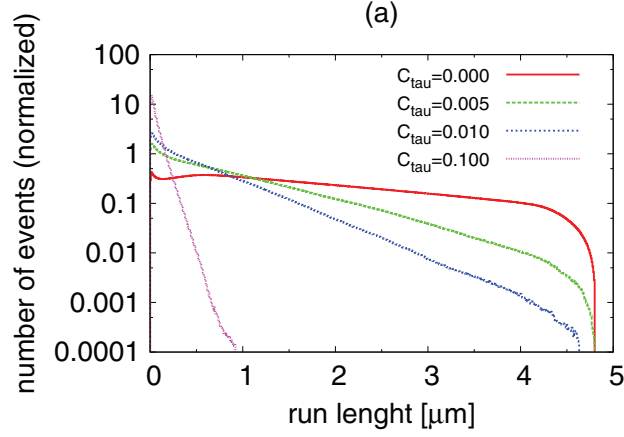

(b)

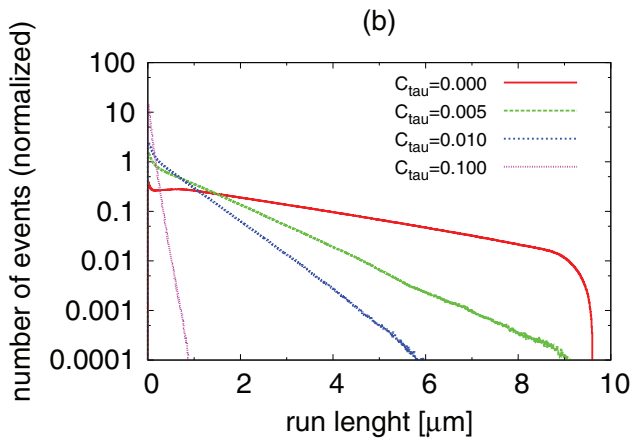

FIG. 6. (Color online) Histograms showing the normalized number of events for the run length of KIF1A motors for several tau concentrations, for two microtubule lengths: (a) $4.8 \mu \mathrm{m}(L=600$ sites $)$, (b) $9.6 \mu \mathrm{m}\left(L=1200\right.$ sites). Model parameters are $p_{a}=0.67$, $p_{m}=0.5, \omega_{d}=\beta_{1}=\beta_{2}=0.0001\left[\mathrm{~ms}^{-1}\right], \omega_{s}=0.145\left[\mathrm{~ms}^{-1}\right], \omega_{f}=0.055\left[\mathrm{~ms}^{-1}\right], \omega_{b}=0.6\left[\mathrm{~ms}^{-1}\right], \gamma_{1}=\gamma_{2}=\delta=0, \omega_{h}=0.2\left[\mathrm{~ms}{ }^{-1}\right]$, and $\omega_{a}=\alpha=0.00001\left[\mathrm{~ms}^{-1}\right]$. The results shown are averages over 1000 realizations of the initial tau distribution for the given tau concentration. The total number of MCSs of each realization is $2 \times 10^{7}$. The thermalization occurs for $2 \times 10^{5}$ MCSs in case (a), and for $2 \times 10^{6}$ MCSs in case (b). 

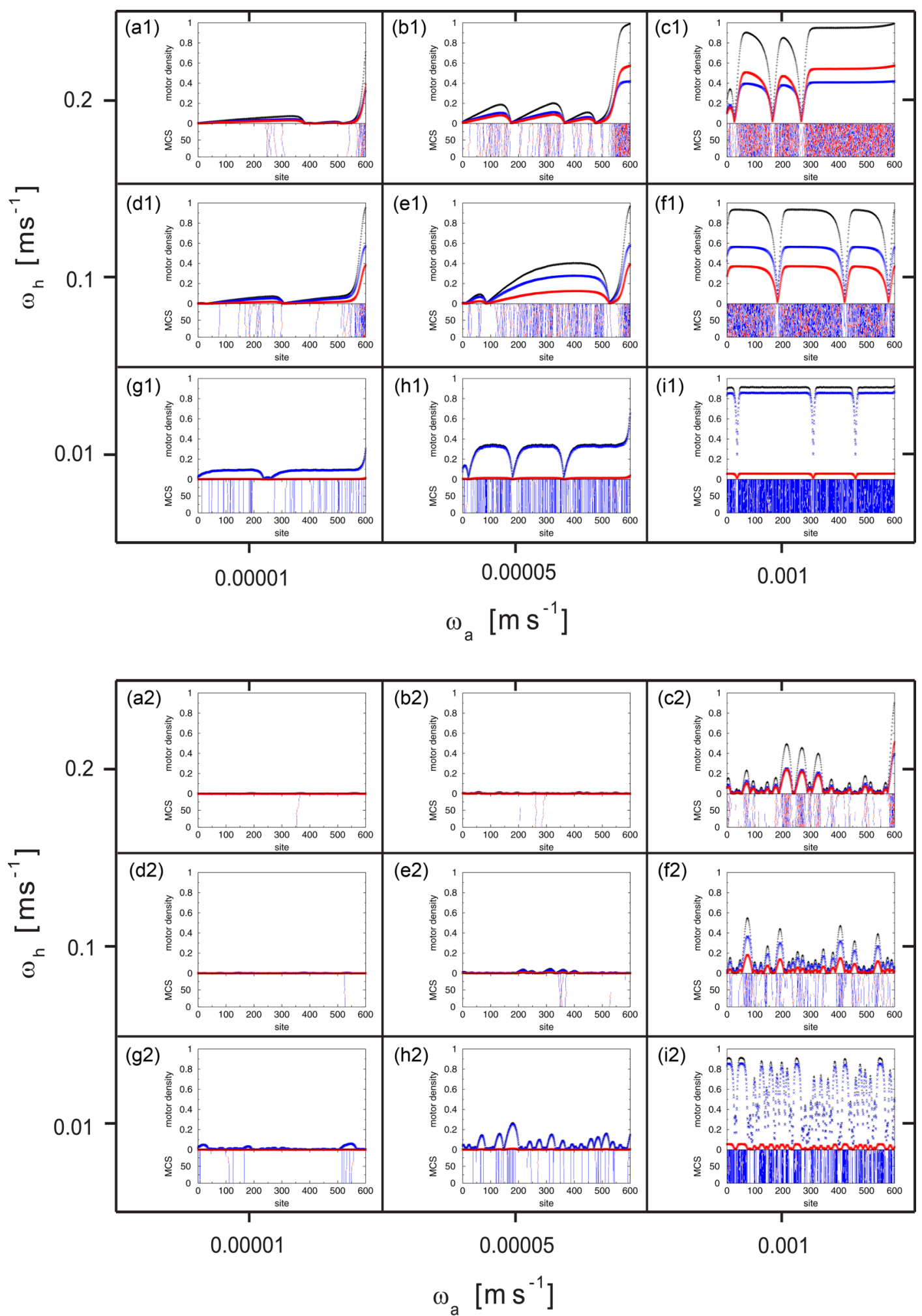

FIG. 7. (Color online) Motor density for different combinations of parameters $\omega_{a}$ (related to KIF1A concentration) and $\omega_{h}$ (related to ATP concentration). The upper figure corresponds to a low-tau concentration, $C_{\mathrm{tau}}=0.005$, and the lower figure, to high-tau concentration, $C_{\text {tau }}=0.1$. The stationary state of the filament depends crucially on the values of the ATP, KIF1A, and tau concentrations. For each combination of parameters, we show the total motor density in black, the state 1 motor density in blue (dark gray) and the state 2 motor density in red (light gray). Each row of the space plot at the bottom of each figure represents the state of the filament for a given specific time. One hundred MCSs, with the system in the stationary state, are displayed. A given point of the row is white if the associated site was empty at that time, blue (dark gray) if it was occupied with a motor in state 1 , and red (light gray) if it was occupied with a motor in state 2 . The other model parameters are equal for all cases: $L=600, \omega_{d}=\beta_{1}=\beta_{2}=0.0001\left[\mathrm{~ms}^{-1}\right], \omega_{f}=0.055\left[\mathrm{~ms}^{-1}\right], \omega_{s}=0.145\left[\mathrm{~ms}^{-1}\right], \omega_{b}=0.6\left[\mathrm{~ms}^{-1}\right], \gamma_{1}=\gamma_{2}=\delta=0$, and $\alpha=\omega_{a}$. 
Figure 6 shows the differential effect of several tau concentrations on the KIF1A run length distributions for two MT lengths. Figure 6(a) corresponds to a MT of $4.8 \mu \mathrm{m}$ (600 sites), and Fig. 6(b) corresponds to a MT of $9.6 \mu \mathrm{m}$ (1200 sites). In the same way as in Figs. 4 and 5 the results shown correspond to averages over 1000 simulations with the same parameters except for the random generator seed. This means that the tau concentration is the same but the specific tau distribution changes (that is, the sites where there are tau molecules in each case). Note that these results agree well with the communicated experimental observations [9], in which the distributions move towards smaller run lengths for larger tau concentrations. We have included Fig. 6(b), in which the MT has a length of $9.6 \mu \mathrm{m}$, in order to make a direct comparison with those results.

Figure 7 shows a phase diagram in the $\omega_{a}-\omega_{h}$ plane as is done by earlier investigators working with KIF1A models $[17,22,23]$. In the upper part of the figure we show a case corresponding to low-tau concentration $\left(C_{\text {tau }}=0.005\right)$, whereas in the bottom part of the figure we show a high-tau concentration case $\left(C_{\mathrm{tau}}=0.1\right)$.

For low-tau concentration, when the motor concentration is high $\left(\omega_{a}=0.001 \mathrm{~ms}^{-1}\right)$, we have a high motor density stationary state, Figs. 7(c1), 7(f1), and 7(i1). In these cases the value of $\omega_{h}$ regulates only the fraction of motors in each state, as can be noted looking at the density profiles. For the cases in Figs. 7(g1), and 7(h1) we have a low-density stationary state, whereas in the remaining cases [corresponding to Figs. 7(a1), 7(b1), 7(d1), and 7(e1)] we have phase coexistence with a high-density region near the plus end and a low-density region extending towards the minus end. Note the abrupt increase in the motor density for these cases.

For high-tau concentration, the tau-KIF1A interaction guarantees a low motor density stationary state for most of the cases [Figs. 7(a2), 7(b2), 7(d2), 7(e2), 7(g2), and 7(h2)]. In this case, in which there are many tau molecules, the effect of tau preventing motor attachments and forcing moving motors to detach results in low motor density stationary states for all cases but those of high motor concentrations [Figs. 7(c2), 7(f2), and 7(i2)]. It is worth noting that there exists a high enough tau concentration such that leads to a low motor density stationary states for all cases considered here. Thus, we found that the stationary state dynamics of the system depends crucially on tau concentration, and it is not enough to characterize it in terms of $\omega_{a}$ and $\omega_{h}$, as has been done previously [17,22,23].

\section{CONCLUSIONS}

In this paper we have presented a stochastic model for the intracellular transport by KIF1A motor proteins that takes into account the effect of the MAP tau. Our model is an extension of the model of Nishinari et al. for the trafficking of KIF1A without tau, and it was our intention to preserve one of its virtues, that is, to relate the properties of the transport to experimentally controllable quantities. In this way we model the effect of one tau molecule by means of two parameters, $p_{a}$ and $p_{m}$. The effect the tau molecule has in preventing kinesin motor binding to MT is characterized by $p_{a}$, and the effect it has forcing moving motor proteins to detach from the MT upon an encounter by $p_{m}$. The values of these parameters have been determined from obtained experimental results [9] and have proven to be robust from the point of view of the simulation. That is, small changes on the values of these parameters do not modify the qualitative behavior of the observables discussed here. Moreover, changes in orders of magnitude on the parameters $p_{a}$ and $p_{m}$, change the value of the observables in the same order of magnitude (results not shown).

Our model coincides with the model [17] in the limit where no tau molecules are present in the filament, and the same is true for the analytical approximate high-density results shown in Eqs. (9) and (10).

Our results agree well with the communicated experimental observations Ref. [9]; that is, tau decreases the binding frequency and run length of the motors. Moreover, we found a qualitative agreement with the run length distribution found by those authors.

The processivity of molecular motors, which is crucial for the intracellular transport, can be defined in several ways [24], two of which being the attachment lifetime of the motor proteins in the filament (bound time) and the mean length traveled by the motor proteins in a single run in the filament (run length). We found significant effects on both bound time and run length of KIF1A motors due to the presence of bound tau molecules in the MT. Furthermore, tau concentration together with ATP concentration and motor concentration are the crucial quantities that determine the stationary state of the system. In this way, the results shown here not only establish the importance of tau in critical magnitudes associated with the intracellular transport by kinesins, but also suggest that tau could play an important role in its regulation. Neurons could locally regulate tau concentration in order to prevent jamming and guarantee a normal intracellular transport. This regulation could be both in time and in space. In this last case, we can think of a gradient of tau concentration growing towards the axon terminal. A small tau concentration near the cell body would not interfere with kinesin binding to MTs, whereas a large tau concentration near the axon terminal would facilitate the cargo release after it has been transported by the kinesin along the axon.

\section{ACKNOWLEDGMENTS}

The authors thank Adolfo J. Banchio for fruitful discussions. J.S. and P.W.L. acknowledge financial support by SECyT-UNC (Argentina).

\footnotetext{
[1] J. Howard, Mechanics of Motor Proteins and the Cytoskeleton (Sinauer Associates, Sunderland, MA, 2001).

[2] M. Aridor and L. A. Hannan, Traffic 1, 836 (2000).
}

[3] I. Toyoshima, K. Kato, M. Sugawara, C. Wada, and O. Masamune, Neurosci. Lett. 249, 103 (1998).

[4] G. Lee, Curr. Opin. Cell Biol. 5, 88 (1993).

[5] N. Hirokawa, Curr. Opin. Cell Biol. 6, 74 (1994). 
[6] S. C. Papasozomenos and L. I. Binder, Cell Motil. Cytoskeleton 8, 210 (1987).

[7] A. Ebneth, R. Godemann, K. Stamer, S. Illenberger, B. Trinczek, E.-M. Mandelkow, and E. Mandelkow, J. Cell Biol. 143, 777 (1998).

[8] B. Trinczek, A. Ebneth, E.-M. Mandelkow, and E. Mandelkow, J. Cell Sci. 112, 2355 (1999).

[9] R. Dixit, J. L. Ross, Y. E. Goldman, and E. L. F. Holzbaur, Science 319, 1086 (2008).

[10] B. S. Govindan, M. Gopalakrishnan, and D. Chowdhury, Europhys. Lett. 83, 40006 (2008).

[11] V. Belitsky and G. M. Schütz, J. Stat. Mech.: Theor. Exp. (2011) P07007.

[12] A. Melbinger, L. Reese, and E. Frey, Phys. Rev. Lett. 108, 258104 (2012).

[13] D. Johann, C. Erlenkämper, and K. Kruse, Phys. Rev. Lett. 108, 258103 (2012).

[14] B. Derrida, Phys. Rep. 301, 65 (1998).

[15] A. Parmeggiani, T. Franosch, and E. Frey, Phys. Rev. Lett. 90, 086601 (2003).
[16] N. Hirokawa, R. Nitta, and Y. Okada, Nat. Rev. Mol. Cell Biol. 10, 877 (2009).

[17] K. Nishinari, Y. Okada, A. Schadschneider, and D. Chowdhury, Phys. Rev. Lett. 95, 118101 (2005).

[18] C. Leduc, K. Padberg-Gehle, V. Varga, D. Helbing, S. Diez, and J. Howard, Proc. Natl. Acad. Sci. USA 109, 6100 (2012).

[19] P. Greulich and A. Schadschneider, Phys. Rev. E 79, 031107 (2009).

[20] Y. Chai, R. Lipowsky, and S. Klumpp, J. Stat. Phys. 135, 241 (2009).

[21] F. Turci, A. Parmeggiani, E. Pitard, M. C. Romano, and L. Ciandrini, Phys. Rev. E 87, 012705 (2013).

[22] J. Sparacino, P. W. Lamberti, and C. M. Arizmendi, Phys. Rev. E 84, 041907 (2011).

[23] P. Greulich, A. Garai, K. Nishinari, A. Schadschneider, and D. Chowdhury, Phys. Rev. E 75, 041905 (2007).

[24] A. Parmeggiani, F. Jülicher, L. Peliti, and J. Prost, Europhys. Lett. 56, 603 (2001). 